EESTI NSV TEADUSTE AKADEEMIA TOIMETISED. 28. KOIDE

FOOSIKA * MATEMAATIKA. 1979, NR. 2

ИЗВЕСТИЯ АКАДЕМИИ НАУК ЭСТОНСКОН ССР. ТОМ 28 ФИЗИКА * МАТЕМАТИКА. 1979, № 2

B. ОЛЬМАН

\title{
МИНИМАКСНОЕ ДОВЕРИТЕЛЬНОЕ ОЦЕНИВАНИЕ ПАРАМЕТРА СДВИГА
}

\author{
V. OLMAN. NIHKEPARAMEETRI USALDATAVUSE MINIMAKSHINNANG \\ V. OLMAN. A MINIMAX CONFIDENCE ESTIMATION OF LOCATION PARAMETER
}

(Представлена Н. Алумяэ)

Рассмотрим стохастическую модель

$$
\mathbf{Y}=\theta+\mathcal{E}, \quad E \mathcal{E}=\mathbf{0},
$$

где $\mathbf{Y}$ и $\varepsilon-$ случайные $m$-мерные векторы, $\theta-$ неизвестный неслучайный $m$-мерный вектор. Задача заключается в построении минимаксного доверительного множества для оценивания $\theta$ при наблюденном векторе Y. В отличие от $\left[{ }^{1-3}\right]$, где решались аналогичные задачи, здесь используется априорная информация о принадлежности вектора $\theta$ некоторому множеству.

Предположим априори известным, что вектор $\theta$ принадлежит шару $K(r)$ радиуса $r$, т. е. $\theta^{\mathrm{T}} \theta \leqslant r^{2}$, где $r-$ заданная величина. Ограничимся рассмотрением доверительных множеств, представимых эллипсоидами, центрированными линейными статистиками

$$
N(k, \mathbf{u}, \mathbf{D})=\left\{\theta \in R^{m}:(\theta-k \mathbf{Y}-\mathbf{u})^{\mathrm{T}} \mathbf{D}(\theta-k \mathbf{Y}-\mathbf{u}) \leqslant 1\right\},
$$

где $k \in R^{1}, \mathbf{u} \in R^{m}$, а $\mathbf{D}-$ неотрицательно определенная матрица порядка $(m \times m)$.

Определим качество доверительного оценивания величиной гарантированной вероятности $q(k, \mathbf{u}, \mathbf{D})$ накрытия неизвестного вектора $\theta$ случайным эллипсоидом $N(k, \mathbf{u}, \mathbf{D})$, т. е.

$$
q(k, \mathbf{u}, \mathbf{D})=\min _{\theta \in K(r)} P\{\theta \in N(k, \mathbf{u}, \mathbf{D}) \mid \theta\} .
$$

Обозначим через $M_{\gamma}$ класс квадратных матриц D таких, что лебеговская мера эллипсоида $\mu(N(k, \mathbf{u}, \mathbf{D}))$ в пространстве параметра $\theta$ удовлетворяет ограничению

$$
\mu(N(k, \mathbf{u}, \mathbf{D})) \leqslant \gamma .
$$

Тогда задача минимаксного оценивания запишется в виде

$$
\max _{\mathbf{D} \in M_{\gamma^{\prime}} \in R^{m}, k \in R^{1}} q(k, \mathbf{u}, \mathbf{D}) .
$$


Т е о рем а. Нусть распределение вектора $\varepsilon$ в модели (1) сферически симметрично, т. е. функция плотности этого вектора $f(\varepsilon)=$ $=g\left(\varepsilon^{\mathrm{T}} \mathcal{E}\right)$, причем $g(t), t \in(0, \infty)$, выпуклая функция. Тогда задача (2) сводится к решению одномерной экстремальной задачи

$$
\max _{h \in R^{1}} \int_{\left(e_{1}-(k-1) r\right)^{2}+\sum_{i=2}^{m} e_{t}^{2} \leqslant c^{-2} h^{2}} g\left(\mathbf{e}^{\mathrm{T}} \mathbf{e}\right) d \mathbf{e},
$$

причем оптимальными являются $\mathbf{u}_{0}=\mathbf{0} u \mathbf{D}_{0}=c \mathbf{I}_{m}\left(\mathbf{I}_{m}-\right.$ единичная матрица порядка $(m \times m)), \mathbf{D}_{0} \in M_{\gamma}$.

Д ок а з а тельс т в о. Перепишем функцию $q(k, \mathbf{u}, \mathbf{D})$ в интегральном виде

$$
q(k, \mathbf{u}, \mathbf{D})=\min _{\theta \in K(r)} \int_{H_{1}} f(\mathbf{y}-\theta) d \mathbf{y}=\min _{\theta \in K(r) H} \int_{H} g\left(\mathbf{e}^{\mathrm{T}} \mathbf{e}\right) d \mathbf{e},
$$

где $H_{1}=\left\{\mathbf{y} \in R^{m}:(\theta-k \mathbf{y}-\mathbf{u})^{\mathrm{T}} \mathbf{D}(\theta-k \mathbf{y}-\mathbf{u}) \leqslant 1\right\}$,

$$
H_{2}=\left\{\mathrm{e} \in R^{m}:((1-k) \theta-\mathbf{u}-k \mathrm{e})^{\mathrm{T}} \mathbf{D}((1-k) \theta-\mathbf{u}-k \mathrm{e}) \leqslant 1\right\} .
$$

Пусть $0 \leqslant \lambda_{1}{ }^{2} \leqslant \lambda_{2}{ }^{2} \leqslant \ldots \leqslant \lambda_{m}{ }^{2}-$ собственные числа матрицы D. Очевидно, случай $k=1$ соответствует $r=\infty$. Обозначим через $\theta_{0} \in K(r)$ вектор такой, что $\mathbf{V}_{\mathbf{u}}=(1-k) \theta_{0}-\mathbf{u}-$ собственный вектор матрицы D, соответствующий ее максимальному собственному числу, причем $(1-k) \theta_{0}{ }^{\mathrm{T}} \mathbf{u}<0(k \neq 1)$. Тогда

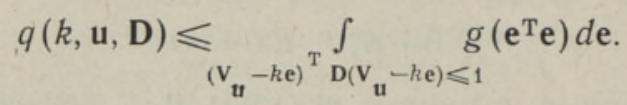

В правой части неравенства (4) произведем ортогональную замену переменных $\mathrm{Ce}=\vec{\eta}$ так, чтобы $\mathrm{CDC}^{\mathrm{T}}=\Lambda$ стала диагональной матрицей с элементами $\lambda_{1}{ }^{2}, \lambda_{2}{ }^{2}, \ldots, \lambda_{m}^{2}$ на главной диагонали. Получаем

$$
q(k, \mathbf{u}, \mathbf{D}) \leqslant \int_{G} g\left(\sum_{i=1}^{m-1} \eta_{i}^{2}+\eta_{m}^{2}\right) \overrightarrow{d \eta}
$$

где $\quad G=\left\{\left(\eta_{1}, \ldots, \eta_{m}\right) \in R^{m}: k^{2} \sum_{i=1}^{m-1} \lambda_{i}^{2} \eta_{i}^{2}+\lambda_{m}^{2}\left(\left\|\mathbf{V}_{\text {u }}\right\|-k \eta_{m}\right)^{2} \leqslant 1\right\}$. Ho

в силу выбора вектора $\theta$ имеем

$$
\left\|\mathbf{V}_{\mathbf{u}}\right\|^{2}=(1-k)^{2} \theta_{0}^{\mathrm{T}} \theta_{0}-2(1-k) \mathbf{u}^{\mathrm{T}} \boldsymbol{\theta}_{0}+\mathbf{u}^{\mathrm{T}} \mathbf{u} \geqslant(1-k)^{2} r^{2}
$$

и, следовательно (см. лемму 1 из $\left.\left[{ }^{4}\right]\right)$, правая часть неравенства (5) мажорируется величиной

$$
\int_{G_{0}} g\left(\sum_{i=1}^{m-1} \eta_{i}^{2}+\eta_{m}^{2}\right) \overrightarrow{d \eta}
$$

где $\quad G_{0}=\left\{\left(\eta_{1}, \ldots, \eta_{m}\right) \in R^{m}: k^{2} \sum_{i=1}^{m-1} \lambda_{i}^{2} \eta_{i}^{2}+\lambda_{m}^{2}\left((1-k) r-k \eta_{m}\right)^{2} \leqslant 1\right\}$.

Используя лемму, сформулированную и доказанную в конце работы, получаем

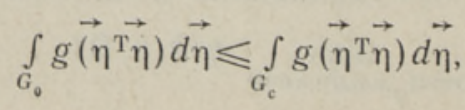


где $G_{c}=\left\{\left(\eta_{1}, \ldots, \eta_{m}\right) \in R^{m}: c^{2}\left(k^{2} \sum_{i=1}^{m-1} \eta_{i}^{2}+\left(k \eta_{m}-(1-k) r\right)^{2}\right) \leqslant 1\right\}$, причем $c^{m}=\prod_{i=1}^{m} \lambda_{i}$

т. е. лебеговские объемы эллипсоидов $N(k, \mathbf{O}, \mathbf{D})$ и $N\left(k, \mathbf{O}, c^{2} \mathbf{I}_{m}\right)$ совпадают. Окончательно, в силу неравенств (5) и (6) получаем

$$
\max _{k, \mathbf{u}, \mathbf{D}} q(k, \mathbf{u}, \mathbf{D}) \leqslant \max _{k \in R^{1}} \int_{G_{\mathrm{c}}} g\left(\eta^{\mathrm{T}} \eta\right) d \vec{\eta} .
$$

Подстановка параметров $\mathbf{u}=\mathbf{0}$ и $\mathbf{D}=c^{2} \mathbf{I}_{m}$ в функцию $q(k, \mathbf{u}, \mathbf{D})$ обращает неравенство (7) в равенство и, следовательно, задача (2) сводится к решению одномерной экстремальной задачи (3), что и дока. зывает теорему.

Л е м м а. Пусть $N_{\alpha}(a, b)=\left\{(x, y) \in R^{2}:(x-\alpha)^{2} / a^{2}+y^{2} / b^{2} \leqslant 1\right\}$, T. е. $N_{\alpha}(a, b)$ - эллипс. Тогда для любой выпуклой на $(0, \infty)$ функции $h(t)$ при $\alpha \in R^{1} u b>a$ имеем

$$
\int_{\alpha_{\alpha}(a, b)} h\left(x^{2}+y^{2}\right) d x d y \leqslant \int_{\alpha^{\left(a_{0}, a_{0}\right)}} h\left(x^{2}+y^{2}\right) d x d y,
$$

где $N_{\alpha}\left(a_{0}, a_{0}\right)$ - круг, лебеговская мера которого совпадает с лебеговской мерой эллипса $N_{\alpha}(a, b)$, , . е. $a_{0}=\sqrt{a b}$ :

$$
\mu\left(N_{\alpha}\left(a_{0}, a_{0}\right)\right)=\mu\left(N_{\alpha}(a, b)\right) .
$$

Д ок а з а тел ь с в о. Введем обозначения

$$
\begin{aligned}
& A_{1}=\left\{(x, y) \in N_{0}\left(a_{0}, a_{0}\right) \backslash\left[N_{0}\left(a_{0}, a_{0}\right) \cap N_{0}(a, b)\right], x \geqslant 0, y \geqslant 0\right\}, \\
& A_{2}=\left\{(x, y) \in N_{0}(a, b) \backslash\left[N_{0}\left(a_{0}, a_{0}\right) \cap N_{0}(a, b)\right], x \geqslant 0, y \geqslant 0\right\} .
\end{aligned}
$$

Нетрудно заметить, что в силу (8)

$$
\mu\left(A_{1}\right)=\mu\left(A_{2}\right) .
$$

Используя симметрию эллипса относительно оси $O Y$, получаем

$$
\begin{aligned}
& \int_{\alpha^{\left(a_{0}, a_{0}\right)}} h\left(x^{2}+y^{2}\right) d x d y-\int_{\alpha^{(a, b)}} h\left(x^{2}+y^{2}\right) d x d y= \\
= & 2\left[\int_{A_{1}}\left(h\left[(x+\alpha)^{2}+y^{2}\right]+h\left[(x-\alpha)^{2}+y^{2}\right]\right) d x d y-\right. \\
& \left.-\int_{A_{2}}\left(h\left[(x+\alpha)^{2}+y^{2}\right]+h\left[(x-\alpha)^{2}+y^{2}\right]\right) d x d y\right] .
\end{aligned}
$$

Учитывая теорему о среднем и соотношение (9), перепишем равенство (10) в виде

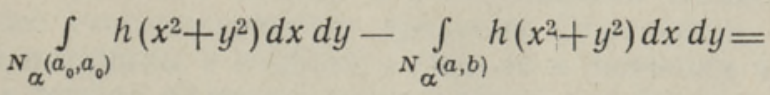

$$
\begin{aligned}
& =2 \mu\left(A_{1}\right)\left[h\left(\left(x_{1}+\alpha\right)^{2}+y_{1}^{2}\right)+h\left(\left(x_{1}-\alpha\right)^{2}+y_{1}^{2}\right)-\right. \\
& \left.-h\left(\left(x_{2}+\alpha\right)^{2}+y_{2}^{2}\right)-h\left(\left(x_{2}-\alpha\right)^{2}+y_{2}^{2}\right)\right],
\end{aligned}
$$

где $\left(x_{1}, y_{1}\right) \in A_{1}, \quad\left(x_{2}, y_{2}\right) \in A_{2}$. По определению множеств $A_{1}$ и $A_{2}$ получаем $x_{1}^{2}+y_{1}^{2}<x_{2}^{2}+y_{2}^{2}$ и $x_{1}>x_{2} \geqslant 0$ и, следовательно, в силу 
выпуклости функции $h(\cdot)$, правая часть равенства (11) положительна, что и доказывает лемму.

С ле д с т в и е. Пусть $N(\Lambda, 1)=\left\{\mathrm{x} \in R^{m}:(\mathrm{x}-1)^{\mathrm{T}} \Lambda(\mathrm{x}-1) \leqslant 1\right\}$, где $\Lambda$ - диагональная матрица с элементами $\lambda_{1}{ }^{2}, \lambda_{2}{ }^{2}, \ldots, \lambda_{m}{ }^{2}$ на главной диагонали, а $1^{\mathrm{T}}=(\alpha, 0, \ldots, 0)$. Тогда для любой выпуклой на $(0, \infty)$ функциии $h(t)$, любого $\alpha \in R^{1} u \lambda_{1} \geqslant \min _{1 \leqslant i \leqslant m} \lambda_{i}$ справедливо

$$
\int_{N\left(\lambda, \mathbf{I}_{m}, 1\right)} h\left(\mathbf{x}^{\mathrm{T}} \mathbf{x}\right) d \mathbf{x} \geqslant \int_{N(\lambda, 1)} h\left(\mathbf{x}^{\mathrm{T}} \mathbf{x}\right) d \mathbf{x},
$$

где $\lambda$ выбрано так, что $\lambda^{m}=\operatorname{det}(\Lambda)$, т. е. лебеговские обтемы $N\left(\lambda \mathbf{I}_{m}, 1\right)$ u $N(\Lambda, 1)$ совпадают.

Д о к а з а т е л с т в о осуществляется переходом в (12) к повторному интегрированию и использованием леммы.

\section{ЛИТЕРА Т У Р А}

1. А л е й н е р Р. Ш., Докл. АН СССР, 196, № 5, 999-1001 (1971).

2. A lbert, A., Ann. Math. Statistics, 37, № 6, 1602-1630 (1966):

3. H a rtiga n, I. A., Ann. Math. Statistics, 41, № 6, 1992-1998 (1970).

4. О ль м а н В., Изв. АН ЭССР, Физ. Матем., 23, № 2, 127-134 (1974).

Ннститут кибернетики Академии наук Эстонской ССР
Поступила в редакцию 22/XII 1978 